\title{
El Efecto de la Cobertura del Suelo en la Variación de las Temperaturas Locales; Naranjo, Alajuela, Costa Rica, 2016
}

\section{The Effect of Soil Cover on Local Temperature Variations; Naranjo, Alajuela, Costa Rica, 2016}

\author{
Enzo Barrientos-Ávila \\ Michael Moya-Calderón ${ }^{2}$ \\ Universidad de Costa Rica, Costa Rica
}

http://dx.doi.org/10.15359/rgac.61-2.7

\begin{abstract}
RESUMEN
El estudio pretende relacionar las variaciones en las temperaturas locales en el distrito central del cantón de Naranjo, Alajuela, mediante el uso de imágenes Landsat 8 y Sistemas de Información Geográfica. Se realizó una vectorización de la cobertura del suelo y se compararon las áreas de las diferentes coberturas con las áreas de los rangos de temperatura obtenidos, mostrando una relación entre las temperaturas altas y las zonas urbanas y las temperaturas bajas y las zonas con vegetación. Mostrar las relaciones entre la cobertura y la temperatura es importante para establecer medidas de mitigación y adaptación efectivas ante los cambios climáticos, y así evitar efectos que van asociadas a las zonas urbanas como lo son las islas de calor.
\end{abstract}

Palabras clave: Landsat, cobertura del suelo, temperatura.

1 Estudiante de Bachillerato en Gestión de los Recursos Naturales, Universidad de Costa Rica. Correo electrónico: enzob2093@gmail.com

2 Magister en Desarrollo Sostenible-Conservación Biológica. Docente-investigador en la Universidad de Costa Rica, Sede de Occidente, Sección de Biología. Programa de Investigaciones de Recursos Naturales Sostenibles. Correo electrónico: michael.moya@ucr.ac.cr 


\begin{abstract}
The study aims to relate the variations in local temperatures in the central district of Naranjo, Alajuela, through the use of Landsat 8 images and Geographic Information Systems. A vectorization of the soil cover was carried out, and the areas of the different coverings were compared with the areas of the temperature ranges obtained, showing a relationship between high temperatures and urban areas and low temperatures and areas with vegetation. Showing the relationship between coverage and temperature is important to establish effective mitigation and adaptation measures in the face of climate changes and thus to avoid effects associated with urban areas such as the heat islands.
\end{abstract}

Keywords: Landsat, soil cover, temperature.

\title{
Introducción
}

Los países en vías de desarrollo son vulnerables ante los cambios extremos que se dan en el clima, es posible que el cambio climático aumente la frecuencia y la cantidad de fenómenos meteorológicos radicales, aumentando a su vez los efectos que se asocian a estos. La resiliencia al cambio climático depende de los modelos de desarrollo que se han creado para los países en crecimiento (Mirza 2003, p.1). Aunque el cambio climático es un proceso global, sus raíces son locales, el control del clima en el medio urbano es una labor que se debe tomar en cuenta para la planificación y la gestión ambiental en las ciudades contra los escenarios que presenta el cambio climático (Romero, Irarrázaval, Opazo, Salgado \& Smith 2010, p.36).

La urbanización ha traído consigo algunas consecuencias, como lo son la perdida de áreas verdes, reducción en la captación de gases de carbono, una mayor emisión de contaminantes de la atmosfera, impermeabilización de los suelos y el almacenamiento de calor en las estructuras y superficies, originando las llamadas islas de calor urbanas (Valor et al. 2000, p.1; Grimmond 2007, p.83).

Estudios realizados en Eurasia sugieren que el cambio en el uso/cobertura del suelo tiene un efecto importante en las temperaturas, así mismo muestran evidencias claras de que el efecto de este cambio no afecta de gran manera las temperaturas promedio, en su lugar afectan las temperaturas máximas y mínimas (Li, et al., 2017, p. 66).

En el contexto latinoamericano, esta labor es de gran importancia, debido a que presenta, de manera extraordinaria, un alto nivel de urbanización (United Nations 2009, p.9). Las ciudades evidencian como el desarrollo económico ha propiciado al cambio climático, todos los países 
del mundo siguen el mismo patrón de crecimiento y concentran de forma desordenada la mayor parte de su población en espacios urbanos (Casillas, García, Leyva \& González 2014, p.140).

Este trabajo pretende relacionar la cobertura del suelo con la variación en las temperaturas locales, con el propósito de establecer posibles acciones para la mitigación y adaptación al cambio climático.

\section{Materiales y métodos}

\section{Área de estudio}

Esta contempla el distrito central del cantón de Naranjo en la provincia de Alajuela, Costa Rica (Fig. 1), el cantón como tal no está tomando medidas visibles para la adaptación al cambio climático, como si lo hacen otros cantones de occidente por medio de planes de arborización urbana, además, se toma el distrito central por ser el que posee un mayor grado de urbanización. El cantón de Naranjo se ubica entre las coordenadas $10^{\circ} 06^{\prime} 23$ " latitud norte y $84^{\circ} 23$ '23" longitud oeste, posee una extensión de $126.62 \mathrm{Km}^{2}$, su distrito central tiene una superficie de $16,85 \mathrm{Km}^{2}$ y una altitud que va desde los 840 hasta los $1361 \mathrm{msnm}$ y presenta una temperatura promedio entre 20 y $22{ }^{\circ} \mathrm{C}$ (Municipalidad de Naranjo, 2012, p.4). 


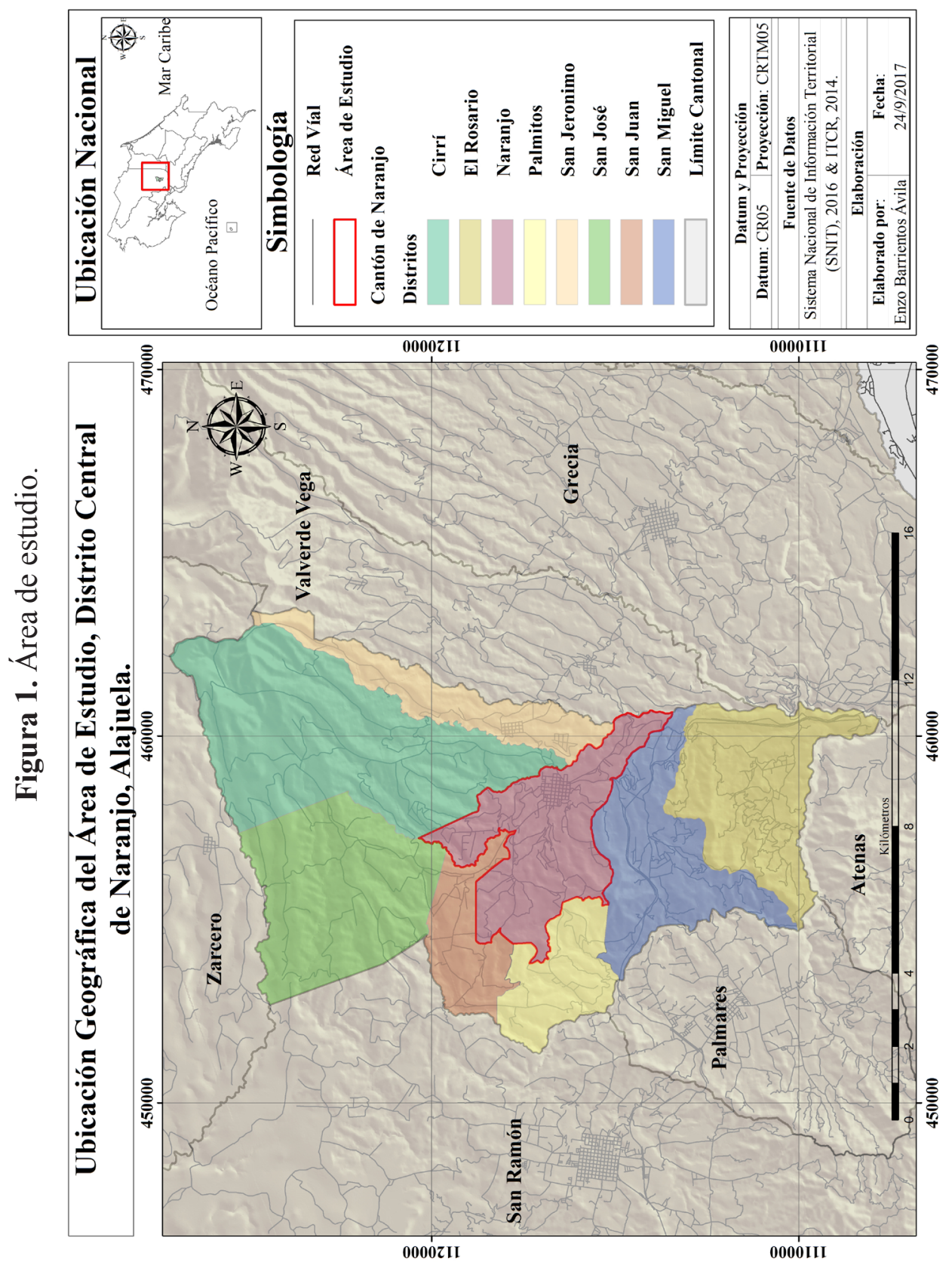


Enzo Barrientos-Ávila, Michael Moya-Calderón. El Efecto de la Cobertura del Suelo en la Variación de las Temperaturas Locales; Naranjo, Alajuela, Costa Rica, 2016

\section{Cobertura del suelo}

Se realizó una vectorización de la fotografía aérea Naranjo del proyecto misión Carta 2005 (Costa Rica Airborne Research and Technology Applications) para determinar los tipos de uso de suelo del distrito por medio del software ARC GIS 10.2.1® (ESRI, 2013). Al resultado de la vectorización, se le agregaron cuadriculas de 1000×1000 m, con la herramienta Cuadrícula Vectorial, del software QGIS 2.14.2 ESSEN® (QGIS Development Team, 2016); dentro de estas cuadriculas se creó un punto en el centro con la herramienta Centroides de Polígonos, dando como resultado 32 puntos, los cuales se visitaron los días 1, 2 y 3 de Agosto de 2016, para realizar comprobación de campo de la exactitud de la vectorización en parcelas de 20x20 m, con el uso de GPS Garmin Oregon 550. Se determinaron las siguientes coberturas:

- Uso urbano: todas aquellas zonas donde se presenta infraestructura urbana, por ejemplo, casas, carreteras, locales comerciales.

- Cobertura forestal: aquellas áreas con presencia de árboles, ya sean naturales o artificiales, que no se encuentren dentro de áreas de cultivos ni en zonas verdes; presentando características más naturales.

- Cultivos con sombra: zonas destinadas para el cultivo, con presencia de árboles en ellas cubriendo el $20 \%$ o más de la plantación.

- Cultivos sin sombra: zonas en las que se desarrolla el cultivo, con poca (menos de $20 \%$ ) o sin presencia de árboles.

- Suelo descubierto: suelo con poca o sin ninguna cobertura de plantas herbáceas.

- Tacotal: terreno no utilizado para el cultivo, cubierto espesamente de plantas herbáceas y arbustos.

- Zona verde arbolada: aquellas zonas que son utilizadas como jardines, parques o zonas de recreación, locales comerciales que cuentan con presencia de árboles.

\section{Estimación de temperaturas}

Para la estimación de las temperaturas se descargaron imágenes Landsat 8 del 4 de octubre del año 2015 de (USGS, 2016) y se analizaron mediante el software QGIS 2.14.2 ESSEN ${ }^{\circledR}$ con el uso del complemento Semi-Automatic Classification (SCP) Congedo (2015) y la metodología establecida por el mismo. 
Se realizaron cruces de cada tipo de cobertura con cada uno de los cinco rangos de temperatura establecidos, mediante la herramienta Intersect (ARC GIS), para establecer el porcentaje de área de cada cobertura que se encontraba dentro de cada rango de temperatura. Para efectos de esta investigación, se establecieron los siguientes rangos de temperatura, según los datos obtenidos del modelo realizado:
1. $24,41-26,76{ }^{\circ} \mathrm{C}$
2. $26,74-28,49^{\circ} \mathrm{C}$
3. $28,50-30,10^{\circ} \mathrm{C}$
4. $30,11-32,16^{\circ} \mathrm{C}$
5. $32,17-35,88^{\circ} \mathrm{C}$

\section{Resultados}

Cobertura del suelo: en total se identificaron siete distintas coberturas de suelo en el distrito central, los cuales se pueden observar en la Figura 2, con su respectiva distribución dentro del área de estudio. La cobertura de suelo con mayor extensión es el cultivo con sombra, seguido del cultivo sin sombra y el uso urbano, con menor extensión se encuentran el tacotal y las zonas verdes arboladas (Cuadro 1). 
Enzo Barrientos-Ávila, Michael Moya-Calderón. El Efecto de la Cobertura del Suelo en la Variación de las Temperaturas Locales; Naranjo, Alajuela, Costa Rica, 2016

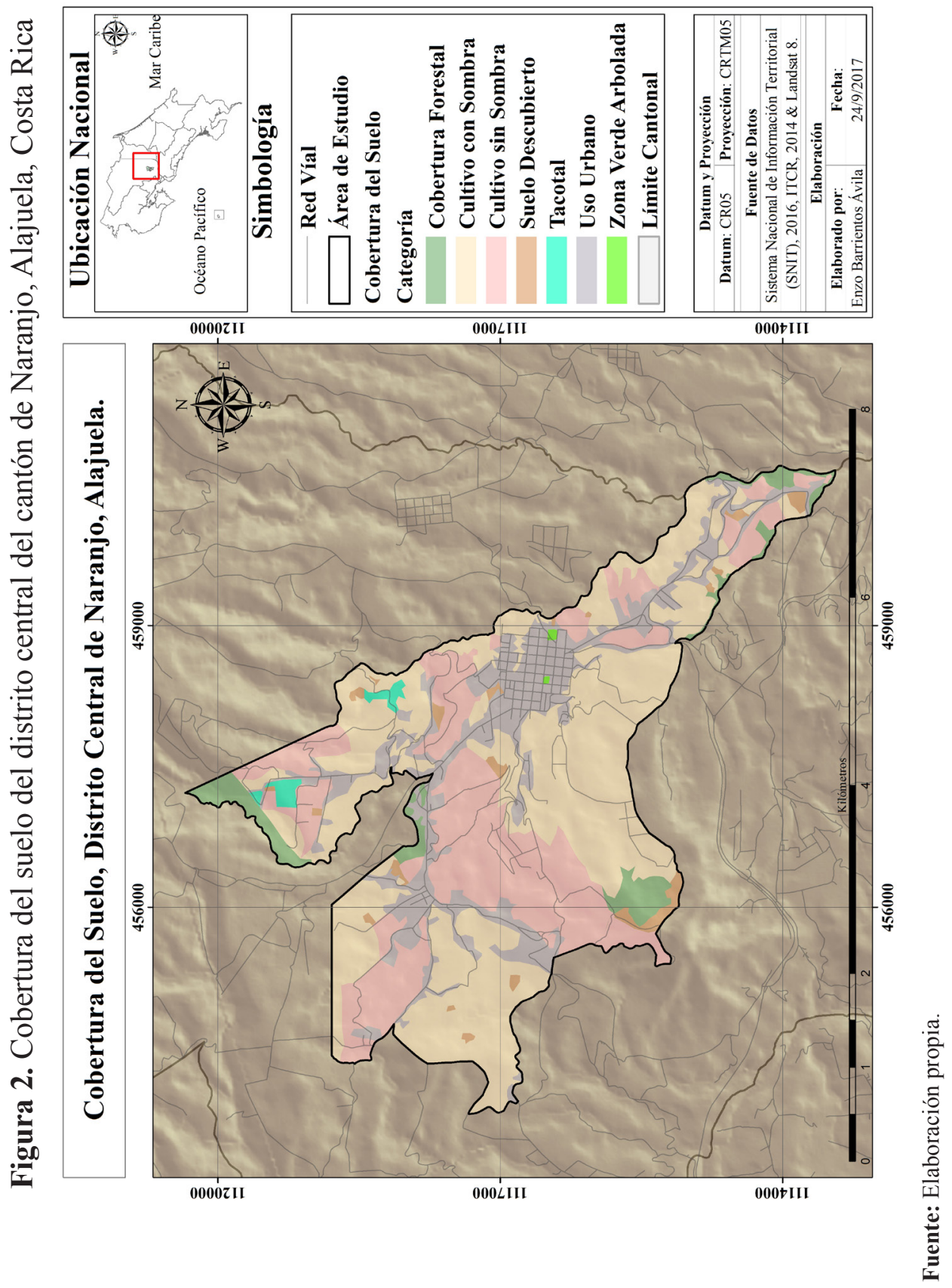


Cuadro 1. Cantidad de área por tipo de cobertura y sus correspondientes porcentajes.

\begin{tabular}{|l|c|c|}
\hline \multicolumn{1}{|c|}{ Categoría } & Área $\left.\mathbf{( m}^{\mathbf{2}}\right)$ & Porcentaje de Área (\%) \\
\hline Cobertura forestal & 812649,65 & 4,82 \\
\hline Cultivo con sombra & 7831042,27 & 46,48 \\
\hline Cultivo sin sombra & 4632109,84 & 27,50 \\
\hline Suelo descubierto & 342503,04 & 2,03 \\
\hline Tacotal & 146513,63 & 0,87 \\
\hline Uso urbano & 3068518,41 & 18,21 \\
\hline Zona verde arbolada & 15907,15 & 0,09 \\
\hline
\end{tabular}

Temperatura: la temperatura más alta es de $35,88^{\circ} \mathrm{C}$ y la más baja de $23,41^{\circ} \mathrm{C}$, en la Figura 3, se observa la distribución de la temperatura dentro del área de estudio, siendo el rango de 28,50 a $30,10{ }^{\circ} \mathrm{C}$ el que presenta mayor distribución dentro del área de estudio y el de 32,17 a 35,88 ${ }^{\circ} \mathrm{C}$ el de menor distribución.

Cuadro 2. Cantidad de área de los rangos de temperatura dentro del área de estudio y su porcentaje de área.

\begin{tabular}{|c|c|c|}
\hline Rango de Temperatura & Área (m2) & Porcentaje de Área (\%) \\
\hline $24,41-26,76^{\circ} \mathrm{C}$ & 1371241,99 & 8,22 \\
\hline $26,74-28,49^{\circ} \mathrm{C}$ & 4710017,69 & 28,25 \\
\hline $28,50-30,10^{\circ} \mathrm{C}$ & 5588470,48 & 32,92 \\
\hline $30,11-32,16^{\circ} \mathrm{C}$ & 3862913,52 & 22,75 \\
\hline $32,17-35,88^{\circ} \mathrm{C}$ & 1310350,65 & 7,86 \\
\hline
\end{tabular}


Enzo Barrientos-Ávila, Michael Moya-Calderón. El Efecto de la Cobertura del Suelo en la Variación de las Temperaturas Locales; Naranjo, Alajuela, Costa Rica, 2016

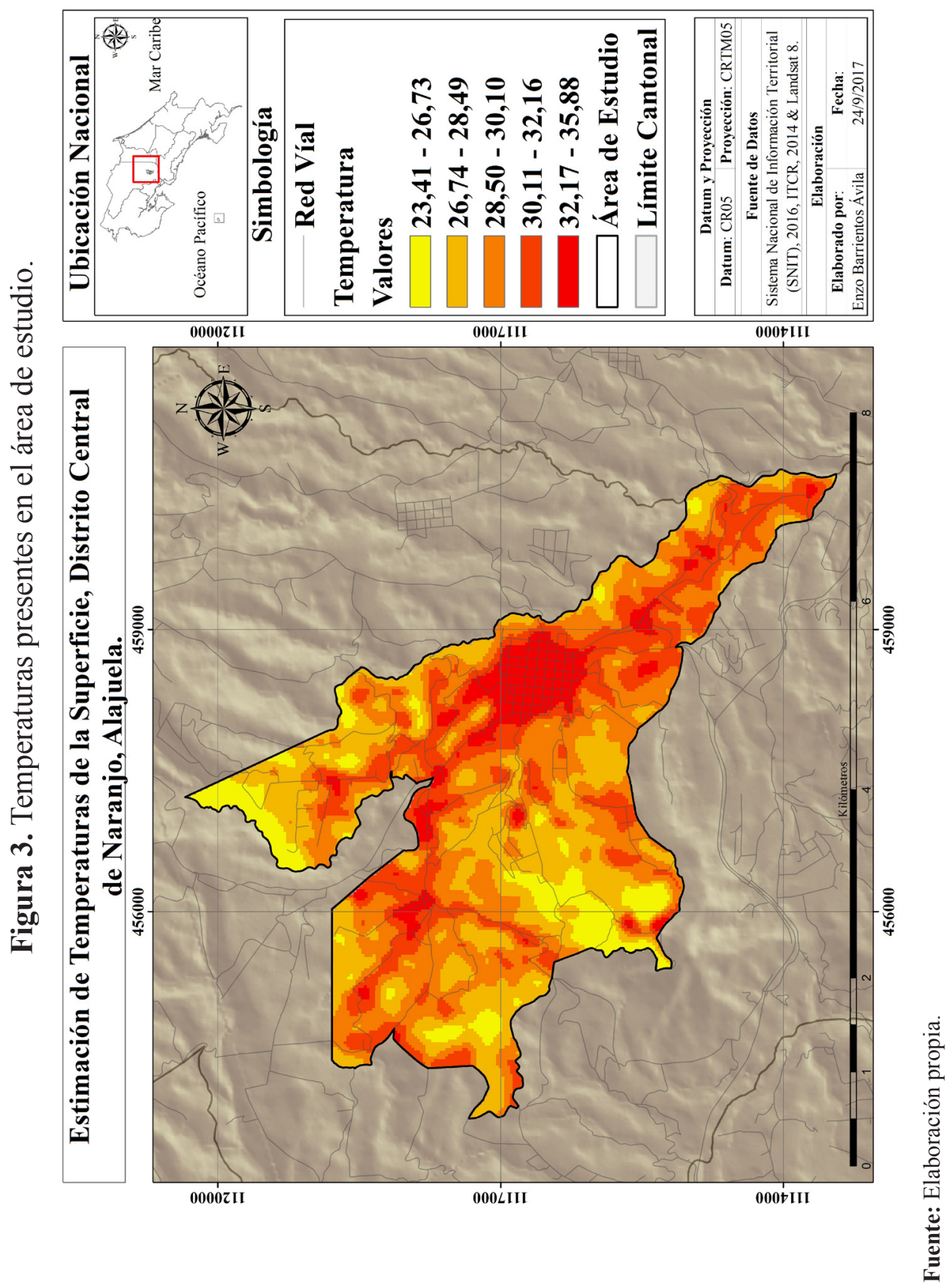


Distribución de la temperatura con respecto a las coberturas de suelo: la cobertura de suelo que presentó una mayor cantidad de área dentro de los rangos de temperatura más altos corresponden al uso urbano y las zonas verdes arboladas, las cuales se encuentran dentro de las zonas urbanas, las cuales poseen la mayor cantidad de su área dentro de los rangos de temperatura de 30,11 a $32,16{ }^{\circ} \mathrm{C}$ y 32,17 a $35,88^{\circ} \mathrm{C}$; por otro lado, en cuanto al rango de temperaturas más bajo, presenta una mayor cantidad de área la cobertura forestal y en los rangos siguientes ambos tipos de cultivos. Las relaciones porcentuales entre área y temperatura se pueden observar en el Cuadro 3.

Cuadro 3. Porcentajes de área presente en cada rango de temperatura, según la cobertura del suelo.

\begin{tabular}{|l|l|l|}
\hline Cobertura del Suelo & \multicolumn{1}{|c|}{ Temperatura $\left({ }^{\circ} \mathbf{C}\right)$} & Porcentaje de Área (\%) \\
\hline Cultivos con sombra & $24,41-26,76$ & 4,75 \\
\cline { 2 - 3 } & $26,74-28,49$ & 36,92 \\
\cline { 2 - 3 } & $28,50-30,10$ & 39,61 \\
\cline { 2 - 3 } & $30,11-32,16$ & 16,00 \\
\cline { 2 - 3 } & $32,17-35,88$ & 2,72 \\
\hline Cultivos sin sombra & $24,41-26,76$ & 12,31 \\
\cline { 2 - 3 } & $26,74-28,49$ & 32,94 \\
\cline { 2 - 3 } & $28,50-30,10$ & 34,82 \\
\hline \multirow{5}{*}{ Uso urbano } & $30,11-32,16$ & 18,30 \\
\cline { 2 - 3 } & $32,17-35,88$ & 1,63 \\
\hline Cobertura forestal & $24,41-26,76$ & 0,31 \\
\cline { 2 - 3 } & $26,74-28,49$ & 1,59 \\
\cline { 2 - 3 } & $28,50-30,10$ & 16,09 \\
\cline { 2 - 3 } & $30,11-32,16$ & 47,49 \\
\cline { 2 - 3 } & $32,17-35,88$ & 34,52 \\
\cline { 2 - 3 } & $24,41-26,76$ & 49,89 \\
\cline { 2 - 3 } & $26,74-28,49$ & 23,55 \\
\cline { 2 - 3 } & $28,50-30,10$ & 16,94 \\
\cline { 2 - 3 } & $30,11-32,16$ & 2,16 \\
\cline { 2 - 3 } & $32,17-35,88$ & \\
\hline
\end{tabular}


Enzo Barrientos-Ávila, Michael Moya-Calderón. El Efecto de la Cobertura del Suelo en la Variación de las Temperaturas Locales; Naranjo, Alajuela, Costa Rica, 2016

\begin{tabular}{|l|l|l|}
\hline Cobertura del Suelo & \multicolumn{1}{|c|}{ Temperatura $\left({ }^{\circ} \mathbf{C}\right)$} & Porcentaje de Área $(\%)$ \\
\hline Suelo descubierto & $24,41-26,76$ & 0,59 \\
\cline { 2 - 3 } & $26,74-28,49$ & 9,26 \\
\cline { 2 - 3 } & $28,50-30,10$ & 30,69 \\
\cline { 2 - 3 } & $30,11-32,16$ & 46,46 \\
\cline { 2 - 3 } & $32,17-35,88$ & 12,99 \\
\hline Tacotal & $24,41-26,76$ & 8,48 \\
\cline { 2 - 3 } & $26,74-28,49$ & 55,26 \\
\cline { 2 - 3 } & $28,50-30,10$ & 29,91 \\
\cline { 2 - 3 } & $30,11-32,16$ & 6,35 \\
\cline { 2 - 3 } & $32,17-35,88$ & 0 \\
\hline Zona verde arbolada & $24,41-26,76$ & 0 \\
\cline { 2 - 3 } & $26,74-28,49$ & 0 \\
\cline { 2 - 3 } & $28,50-30,10$ & 0 \\
\cline { 2 - 3 } & $30,11-32,16$ & 33,75 \\
\cline { 2 - 3 } & $32,17-35,88$ & 66,25 \\
\hline
\end{tabular}

Fuente: Elaboración propia.

\section{Discusión}

Los resultados de la investigación permiten determinar por medio de la distribución de temperaturas dos espacios en el área de estudio: aquel que posee temperaturas más elevadas, el cual se denominará espacio urbano, $\mathrm{y}$, por otro lado, el que posee temperaturas más bajas denominado periferia.

Se observan en el área de estudio variaciones en la temperatura dependiendo del tipo de cobertura del suelo, bajo condiciones naturales sin acciones antrópicas, se podría afirmar que el clima tiene un efecto sobre la distribución de los ecosistemas y de la misma manera, los ecosistemas, por medio de su cobertura pueden afectar los sistemas climáticos (Foley, Heil, Delire, Ramankutty \& Snyder, 2003, p.38). Sin embargo, en el caso de Naranjo se observa una matriz de cobertura de suelo bastante modificada por las diferentes actividades humanas, lo cual no dista de lo acaecido en el oeste del Valle Central, donde se ha dado un proceso de crecimiento de las poblaciones que ha causado que se dé un acercamiento entre ellas, provocando diversidad en la matriz de cobertura (Martínez, 2015, p.26).

Al determinar la cobertura del suelo y las temperaturas en el área de estudio, es posible determinar una estrecha relación entre ambas variables. 
Esto puede obedecer en parte a las propiedades que posee cada cobertura, así, por ejemplo, la vegetación refleja o absorbe gran parte de la radiación solar, dejando que solo pase una pequeña parte hacia el suelo, y en áreas cubiertas por vegetación se refleja la radiación producida en la noche por los suelos de regreso a este, esta característica y la evapotranspiración son las principales enfriadoras del aire, por otra parte, las características de las construcciones hacen que estas almacenen temperatura que liberan en la noche y calientan el aire, además, la cercanía de los edificios y su forma cambia las corrientes de aire (Ochoa, 1999, p. 104 - 105).

La afirmación anterior queda sustentada en los resultados de la investigación, de manera tal, que hay una diferencia de temperaturas marcadas entre las coberturas que poseen algún tipo de vegetación y aquellas que no, como es en su mayoría el espacio urbano. La cobertura que presenta una mayor relación con las temperaturas bajas corresponde a la cobertura forestal, lo cual concuerda con lo planteado por Ochoa $(1999$, p.39), que menciona los efectos de la presencia de vegetación en la regulación del clima, disminuyendo las temperaturas del área en la que está presente. Algunas propiedades como la sombra y procesos de evapotranspiración son las encargadas de regular la temperatura del aire, de modo contrario, al sustituirla por coberturas impermeables, que dirigen el agua directamente a alcantarillados, evitando que se evapore y la humedad en el aire regule la temperatura, se origina el aumento de las temperaturas superficiales y atmosféricas (Environmental Protection Agency [EPA], 2009, p.7).

Es necesario considerar que, no solamente es la cobertura el factor influyente en la temperatura, aspectos como: la presencia o ausencia de las nubes, que van a regular el paso de la radiación solar al suelo, las corrientes de viento, que renuevan constantemente el aire presente en la zona y de la humedad presente también afectan. (Fundación Española para la Ciencia y la Tecnología, 2004, p.15).

A pesar de que solamente se contempló analizar la relación cobertura-temperatura, se puede establecer una relación entre el espacio urbano y la periferia, independientemente, de las variables que podrían haber ocasionado el cambio de la temperatura. Ángel, Ramírez \& Domínguez (2010, p.181) establecen que las interacciones entre urbano-urbano y urbano-rural determinan aumentos o disminuciones en temperatura, la relación urbano-urbano causa incrementos en la temperatura, por esta razón, 
la temperatura se incrementa en el centro de la ciudad y disminuye hacia la periferia. Estás relaciones explican, porque las coberturas de suelo que no presentan una cobertura vegetal densa, pero se encuentran rodeados de usos con coberturas más densas poseen bajas temperaturas.

Aunado a lo anterior, en los resultados también se aprecia que las zonas verdes arboladas dentro del área urbana, en este caso, no parecen tener un efecto regulador del clima, nuevamente Ochoa (1999, p. 39) menciona la ineficiencia en la regulación climática de árboles aislados dentro de espacios urbanos, ya que su efecto desaparece rápidamente por efectos de los movimientos del viento. A pesar de lo anterior, no se deben dejar de hacer esfuerzos por tratar de arborizar las ciudades, ya que un buen plan de arborización que no se quede solo en arboles aislados puede generar muchos beneficios para las ciudades, además de la regulación de temperatura, también mejoran la calidad del aire, mediante la captura de las partículas finas presentes, contribuyen a la reducción del ruido y a la conectividad de espacios naturales dentro y fuera de las ciudades, además de servir como hábitat para especies y mejorar la calidad de vida de las personas (Gutiérrez, 2011, p.4)

\section{Conclusiones}

La cobertura del suelo es un factor influyente en los cambios de temperatura del aire y aunque no es el único factor que determina estos cambios, muestra tener un alto de grado de importancia en estos, dado que las características de las coberturas pueden influenciar otros factores que los determinan. Por ejemplo, las características de las coberturas vegetales colaboran a la presencia de humedad, que colabora en la regulación de temperatura, y, por el contrario, las características de los materiales de las coberturas urbanas inciden en los aumentos de temperatura.

La distribución de las temperaturas divide el área de estudio en dos: en las zonas urbanas se encuentran las temperaturas mayores y en la periferia se encuentran las temperaturas bajas, esto debido a las relaciones que existen entre los espacios urbano-urbano, el cual favorece los aumentos de temperatura y el urbano-rural, que, por otro lado, contribuye a la disminución de la temperatura.

Las temperaturas menores se encuentran asociadas a las coberturas con algún tipo de vegetación, esto se debe a las características que posee la vegetación como su sombra, evapotranspiración, conservación de la 
humedad en el aire, entre otras. Estas características colaboran en la regulación de la temperatura, además de otros beneficios ya mencionados, esta es una de las razones por las que se debe contar con espacios con coberturas con algún tipo de vegetación dentro de las ciudades y no solo arboles aislados, que como ya se mencionó, no causan un efecto significativo en la regulación de temperatura.

\section{Referencias}

Ángel, L., Ramírez, A. \& Domínguez, E. (2010). Isla de calor y cambios espacio-temporales de la temperatura en la ciudad de Bogotá. Rev. Acad. Colomb. Cienc., 34(131), 173-183

Casillas, A., García, R., Leyva, O. \& González, F. (2014). Detección de la Isla Urbana de Calor mediante Modelado Dinámico en Mexicali, B.C., México. Información Tecnología, 25(1), 139-150

Congedo, L. (2016). Semi-Automatic Classification Plugin Documentation. Recuperado de https://media.readthedocs.org.

Environmental Protection Agency (EPA) (2009). Urban Heat Island Basics, Reducing Urban Heat Islands: Compendium of Strategies, Environmental Protection Agency, U.S. Recuperado de https://www.epa.gov.

ESRI. (2013). ArcGIS Desktop: Release 10.2. Redlands, CA: Environmental Systems Research Institute.

Foley, J., Heil, M., Delire, C., Ramankutty, N. \& Snyder, P. (2003). Green surprise? How terrestrial ecosystems could affect earth's climate. Front Ecol Environ, 2003 1(1), 38-44

Fundación Española para la Ciencia y la Tecnología (FECYT). (2004). Meteorología y Climatología: Semana de la Ciencia y la Tecnología 2004. Madrid, España: Universidad Nacional De Educación A Distancia FECYT.

Grimmond, S. (2007). Urbanization and Global Environmental Change: Local Effects of Urban Warming. Geographical Journal, 173(1), 83-88

Gutiérrez, R. (2011). Valor del Arbolado Urbano: La Experiencia de la Municipalidad de Santiago. Mundo Forestal 21, 4-7.

Martínez, T. (2015). Treinta años de Metamorfosis Urbana Territorial en el Valle Central. Informe final para: Vigesimoprimer Informe Estado de la Nación en Desarrollo Humano Sostenible (2014). Programa Estado de la Nación. Recuperado de http://www.estadonacion.or.cr. 
Mirza, M. (2003). Climate Change and Extreme Weather Events: Can Developing Countries Adapt? Climate Policy, 3, 233-248

Municipalidad de Naranjo. (2012). Acta de la Sesión Extraordinaria $N^{o} 02$ del 21 de marzo del 2012. (Actas de la sesión). Recuperado de http:// www.naranjo.go.cr.

Ochoa, J. (1999). La Vegetación como Instrumento para el Control Microclimático. (Tesis inédita de doctorado). Unviersitat Politècnica de Catalunya, Barcelona, España.

QGIS Development Team. (2016). QGIS Geographic Information System. Open Source Geospatial Foundation Project.

Rodríguez, N., Pabón, J., Bernal, N. \& Martínez, J. (2010). Cambio climático y su relación con el uso del suelo en los Andes colombianos. Bogotá, Colombia: Instituto de Investigación de Recursos Biológicos Alexander von Humboldt, Universidad Nacional de Colombia y Departamento Administrativo de Ciencia, Tecnología e Innovación.

Romero, H., Irarrázaval, F., Opazo, D., Salgado, M. \& Smith, P. (2010). Climas Urbanos y Contaminación Atmosférica en Santiago de Chile. EURE, 36(109), 35-62

Sarricolea, P., Aliste, E., Castro, P. \& Escobedo, C. (2008). Análisis de la Máxima Intensidad de la Isla de Calor Urbana Nocturna de la Ciudad de Rancagua (Chile) y sus Factores Explicativos. Revista de Climatología, 8, 71-84

United Nations (2009). World Urbanization Prospects: The 2009 revision. Recuperado de http://ipcc-wg2.gov.

USGS. (2016). United States Geological Survey. Recuperado de https:// www.usgs.gov.

Valor, E., Caselles, V., Coll, C., Sánchez, F., Rubio, E., \& Sospedra, F. (2000). Análisis Comparativo del Efecto de Isla Térmica de la Ciudad de Valencia con Imágenes TM, MUST y AVHRR. Revista de Teledetección, (14), 1-6

Li X, Chen H, Liao H, Hua W, Sun S, Ma H, Li X, Gao C, Zhu S (2017) Potential effects of land cover change on temperature extremes over Eurasia: current versus historical experiments. Int. J. Climatol, $37(1), 59-74$ 